\title{
Linear and nonlinear characterizations of chalcogenide photonic crystal fibers
}

\author{
J. Fatome, C. Fortier, T. N. Nguyen, T. Chartier, F. Smektala, K. Messaad, B. Kibler, S. Pitois, G. \\ Gadret, C. Finot, J. Troles, F. Desevedavy, P. Houizot, G. Renversez, L. Brilland and N. Traynor
}

\begin{abstract}
In this work, we investigate the linear and nonlinear properties of GeSbS and AsSe chalcogenide photonic crystal fibers. Through several experimental set-ups, we have measured the second- and third-order chromatic dispersion, the effective area, losses, birefringence, the nonlinear Kerr coefficient as well as Brillouin and Raman scattering properties.
\end{abstract}

Index Terms-Chalcogenide optical fiber, Microstructured optical fiber, Nonlinear optics.

\section{INTRODUCTION}

$\mathrm{P}$ hotonic crystal fibers (PCFs) are particularly attractive since they offer the possibility to significantly enhance the nonlinearity of the bulk medium by means of a strong light confinement of the electromagnetic field [1]-[2]. Another advantage is the ability to tailor the material dispersion by adjusting the waveguide dispersion design. Consequently, a wide range of applications has strongly benefited from these remarkable features to generate octave-spanning optical supercontinuum [3], for biology [4] or optical metrology [5], to process nonlinear effects with high efficiency and compactness [6], and even to levitate particles [7]. Nevertheless, silica PFCs have a limited nonlinearity because of the weak nonlinear index of silica and more importantly, their intrinsic losses ban any application beyond $2 \mu \mathrm{m}$. Nonsilica glass PCFs have therefore to be considered in order to enhance the field of applications of microstructured optical fibers [8]-[20]. Several glasses have been found to be very

Manuscript received October 29, 2008. (Write the date on which you submitted your paper for review.) This work was supported in part by the Agence National de la Recherche (FUTUR project) and the Conseil Regional de Bourgogne.

J. Fatome, C. Fortier, B. Kibler, S. Pitois, G. Gadret, C. Finot and F. Smektala are with the Institut Carnot de Bourgogne, UMR 5209 CNRSUniversité de Bourgogne, 9 Av. Alain Savary, BP 47870, 21078 Dijon, France (corresponding author to provide phone: 0033(0)380395982; fax: 0033(0)380395971; e-mail: jfatome@u-bourgogne.fr).

T. N. Nguyen, K. Messaad and T. Chartier are with UMR CNRS 6082 FOTON, Laboratoire d'Optronique de l'ENSSAT, 6 rue de Kerampont, 22300 Lannion, France (e-mail: chartier@enssat.fr).

J. Troles, F. Desevedavy and P. Houizot are with Laboratoire Verres et Céramiques (LVC), UMR 6226 CNRS-Université de Rennes I, Campus de Beaulieu, 35042 Rennes, France (e-mail: johann.troles@univ-rennes1.fr).

G. Renversez is with the Institut Fresnel, UMR 6133 CNRS-Université Paul Cézanne Aix-Marseille III, Av. Escadrille Normandie-Niémen, 13397 Marseille Cedex 20, France (e-mail : gilles.renversez@fresnel.fr).

L. Brilland and N. Traynor are with PERFOS, 11 rue de Broglie, 22300 Lannion, France (e-mail: ntraynor@perfos.com). attractive for their nonlinear characteristics including Chalcogenide, Bismuth, Tellurite or lead silicate glasses which nonlinear index are over two to three orders of magnitude higher than fused silica [9]-[16]. Despite the fact that the material dispersion of these glasses is often very large around $1.5 \mu \mathrm{m}$, several results have demonstrated that a PCFs design allows to shift significantly the fiber zero-dispersion towards lower wavelengths [10]-[12]. A relevant illustration of powerful nonlinear compactness and PCF dispersion shift of this non silica PCFs was reported in ref. [11] in which a $4-\mu \mathrm{m}$ bandwidth supercontinuum has been generated thanks to a 8$\mathrm{mm}$ long sample of Tellurite PCF. In another hand, Chalcogenide glasses offer one of the strongest glass nonlinearity (up to $500 \mathrm{x}$ silica) and has been studied in several works and applications [13]-[28]. Eggleton and coworkers have recently demonstrated a high compactness 160Gbit/s demultiplexing device and a supercontinuum generation by means of a chalcogenide fiber taper which nonlinearity reaches 80.000 times that of fused silica [13]-[15]. Recently, we have also investigated the Brillouin and Raman properties of a PCF chalcogenide fiber and shown Brillouin and Raman gains 100 and 180 larger than fused silica which underline the potential of this kind of fiber for amplification or slow/fast light applications [17]-[18].

In this work, we review recent progress in the fabrication of chalcogenide microstructured fibers. We will present, for the first time of our knowledge, an extensive set of linear and nonlinear characterizations of three chalcogenide PCFs based on GeSbS and AsSe glasses including second- and third-order chromatic dispersion, effective area, losses, birefringence, nonlinear Kerr coefficient as well as Brillouin and Raman scattering properties.

\section{MicRostructured CHALCOGENIDE FiBERS}

Chalcogenide glasses are based on Sulphur, Selenium, Tellurium and the addition of other elements such as Arsenic, Germanium, Antimony, Gallium... They are well known for their large infrared transmission window as well as for their large non linearity. In this paper, we have focused our attention on three PCFs based on two compositions, which have been chosen for their great stability against crystallisation effect: $\mathrm{Ge}_{15} \mathrm{Sb}_{20} \mathrm{~S}_{65}$ (GeSbS) for two fibers (GeSbS_1 and GeSbS_2) with two different core diameters and $\mathrm{As}_{40} \mathrm{Se}_{60}$ (AsSe). These glasses present any crystallization peaks in classical differential scanning calorimetry measurements (DSC). 
A crucial difficulty in the fabrication of this type of fiber is to control carefully the impurity level of the starting products as well as of the final fiber. Indeed, carbon and water are frequent contaminant, especially in the case of sulphur. They must be removed from the glass batch by a combination of static and dynamic distillations. The contamination by water during the drawing step must also be avoided by using a dry drawing atmosphere. The different process steps have to be conducted at optimised temperatures to avoid any crystallization phenomenon of the glass in the bulk as well as on its surface.

The stack and draw method was used to elaborate the chalcogenide PCFs [21]. First of all, glass rods are realized with typical sizes around 18-cm length and around 12-mm outside diameter, respectively. The first part of the glass rod is used to draw a single index fiber which allows checking the material level of losses. The other part of this rod is then remelted to obtain the glass tube by centrifugation. Tubes around $12 \mathrm{~mm}$ outside diameter, about $5 \mathrm{~mm}$ inside and $12 \mathrm{~cm}$ length are then obtained by the centrifugation of the previous rod. Theses tubes are finally drawn down to obtain capillaries of around $600 \mu \mathrm{m}$ outside diameter. They were then stacked in a hexagonal lattice around a central rod of the same diameter and placed in a larger jacket tube. This jacket tube is collapsed around the microstructure under depression in the furnace of the drawing tower. Parameters of furnace temperature and depression have to be adjusted to prevent any collapse of both capillary and interstitial holes. These different process steps have to be conducted at optimised temperatures to avoid any crystallization phenomenon of the glass in the bulk as well as on its surface. During the fabrication of tubes by centrifugation, the thermal profile is also dramatically important since the glass after its synthesis is reheated from room temperature to a temperature above the glass temperature (Tg). This is necessary to obtain a suitable viscosity for the centrifugation process but very sensitive, since atomic mobility could allow a crystallization. Another critical step is the stretching of the tube, which can lead to an alteration of the surface quality of capillaries inducing diffusion in the final fiber. The contamination by water during the drawing step must also be avoided by using a dry drawing atmosphere.

A preform for each glass composition was realized in the same experimental conditions. During the fibre drawing process, capillary holes and interstitials are maintained under positive pressure in order to obtain fibers with open interstitial holes. Indeed their presence improves greatly the fiber transmission as they enable to diminish the overlap between the guiding electric field and the interface region of capillaries where numerous scattering centres such as bubbles have already been observed [22]. Figure 1 represents the SEM central region of PCFs for both considered glasses.

During the drawing, the variation of geometrical parameters of the fibers (core and holes sizes) doesn't exceed $2 \%$ for several meters. The uniformity of the geometry is controlled after the drawing by microscopy.
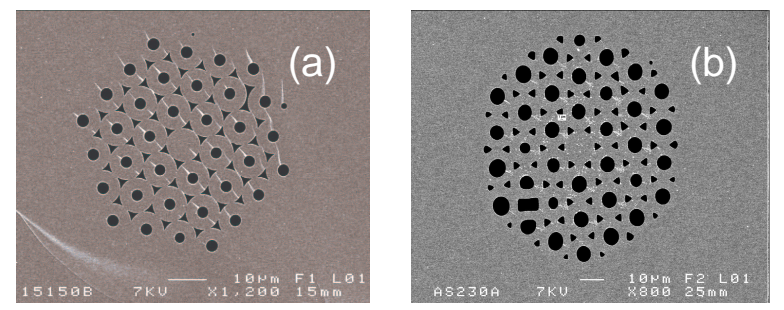

Fig. 1 (a) Cross section of GeSbS_2 PCF (b) Cross section of AsSe PCF.

Table 1 gives, for the three fibers, the average of the geometrical parameters, $\Lambda$ and $\mathrm{d}$. The pitch, $\Lambda$, is the hole to hole distance and $\mathrm{d}$ is the hole diameter. We also report in this table the index of refraction and the transition temperature $\mathrm{Tg}$ for the two kind of glasses. The outer diameter of the GeSbS_1 and AsSe PCF was measured to be $125 \mu \mathrm{m}$ and $280 \mu \mathrm{m}$ for the GeSbS_2 PCF.

\begin{tabular}{|c|c|c|c|c|c|}
\hline Fiber & $\begin{array}{c}\text { Glass } \\
\text { composition }\end{array}$ & $\begin{array}{c}\text { Glass } \\
\text { transition } \\
\text { temperature } \\
T g\left({ }^{\circ} \mathrm{C}\right)\end{array}$ & $\begin{array}{c}\text { Refractive } \\
\text { index at } \\
1,55 \mu \mathrm{m}\end{array}$ & $\begin{array}{c}\Lambda \\
(\mu \mathrm{m})\end{array}$ & $d / \Lambda$ \\
\hline $\mathrm{GeSbS \_ 1}$ & $\mathrm{Ge}_{15} \mathrm{Sb}_{20} \mathrm{~S}_{65}$ & 250 & 2.30 & 9 & 0.31 \\
\hline $\mathrm{GeSbS \_ 2}$ & $\mathrm{Ge}_{15} \mathrm{Sb}_{20} \mathrm{~S}_{65}$ & 250 & 2.30 & 13.25 & 0.31 \\
\hline $\mathrm{AsSe}$ & $\mathrm{As}_{40} \mathrm{Se}_{60}$ & 185 & 2.83 & 7 & 0.42 \\
\hline
\end{tabular}

Table 1: optical and thermal properties of the GeSbS and AsSe PCF fibers.

\section{PROpagation LOSSES}

Propagation losses are of crucial importance for nonlinear phenomena, since they could dramatically reduce the interaction length to a shortest effective length of fiber defined as [29]:

$$
L_{\text {eff }}=\frac{1}{\alpha}[1-\exp (-\alpha L)]
$$

where $L$ is the fiber length and $\alpha$ the linear losses in $\mathrm{m}^{-1}$.

The attenuation losses were first monitored at $1.55 \mu \mathrm{m}$ in the $400 \mu \mathrm{m}$ single index fibers drawn from the initial glass rod and found to be $0.5 \mathrm{~dB} / \mathrm{m}$ for the GeSbS glass and $1.05 \mathrm{~dB} / \mathrm{m}$ for the AsSe [22]. For comparison, the losses in these kinds of multimode fibers can reach $0.1 \mathrm{~dB} / \mathrm{m}$ at $1.55 \mu \mathrm{m}$ [30]. After drawing the PCFs, the propagation losses of the different fibers were then measured at $1550 \mathrm{~nm}$ by means of the so called cut-back technique. Experimental results as well as all optical properties of our three PCF chalcogenide fibers described thereafter are summarized in Table 2, page 6. Any significant change of the physical properties has been observed between the bulk rod and the fibers. But, interfaces defects have been observed between the capillaries in the microstructured [22]. The presence of these defects explained the excess of losses observed in the PCF fibers in comparison with the materials losses. The nature of the defect is not clearly identified. But, it can be bubbles and surface oxidation of the capillaries before the realisation of the stack. Concerning the theoretical guiding losses, in ref. [31], it has been demonstrated that three rings of holes are sufficient to ensure guiding losses below $1 \mathrm{~dB} / \mathrm{m}$. 


\section{EFFECTIVE AREA}

The effective area $A_{\text {eff }}$ was measured at $1550 \mathrm{~nm}$ following a near field method [29]. Using an adequate microscope objective, the intensity at the output of the fiber is recorded on a CCD camera. Note that the camera is calibrated using the near field of a well-known fiber, i.e. a standard single-mode fiber. Figure 2 gives some experimental examples (solid line) of the intensity distribution along several axes of the camera plane for the GeSbS_1 and AsSe fibers. As we can see, both fibers exhibit single mode behaviour. Indeed, for all sections, the output profiles were accurately fitted by means of a Gaussian function and moreover, by changing injection conditions, no any higher order mode was observed even for sections shorter than $10 \mathrm{~cm}$. Finally, the Gaussian fit distributions of Fig. 2 (squares) allow us to determine the mode field diameter $2 w_{0}$ (at 1/e intensity point) and to calculate the effective area thanks to the following formula $A_{e f f}$ $=\pi w_{0}^{2}$.
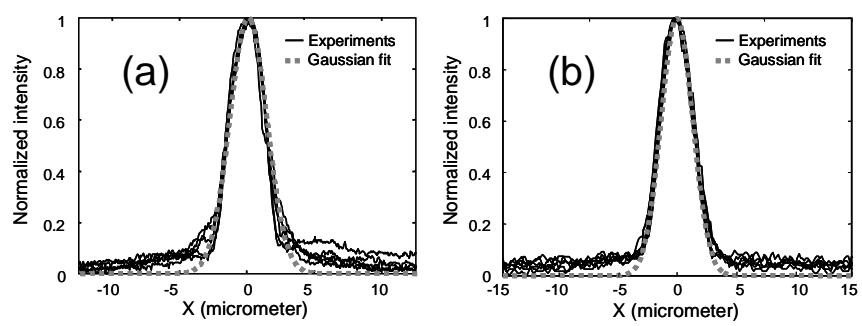

Fig. 2. Experimental traces (solid line) of the intensity distribution in the near field of the GeSbS_1 fiber (a) and the AsSe fiber (b). Squares represent a Gaussian fit distribution.

The measured effective areas of our three PCF chalcogenide fibers are indicated in Table 2.

\section{ChromatiC DISPERSION}

Measuring the chromatic dispersion of short samples of optical fibers with a reasonable accuracy has become of a major interest for the special glass optical fiber designers, manufacturers and researchers. Various methods have been reported in the literature to measure chromatic dispersion [32][35]. For example, in ref. [32] and [33], four wave mixing (FWM) and modulation instability (MI) were proved to be efficient ways to simultaneously measure the Kerr and dispersion coefficients. The variation of the time of flight of short pulses could be also used to measure the chromatic dispersion of sufficiently long fibers. In ref. [34], the authors exploit the higher order soliton compression phenomenon in order to determine the parameters of a silica PCF fiber. But these methods often require a long length of fiber or are only suitable for the anomalous dispersion regime.

In this work, we based our dispersion measurements on an interferometric method which is able to handle short segments of optical fiber [35]. The home-made interferometric set-up is illustrated in Fig. 3 and was based on a Mach-Zehnder interferometer designed with all-fibered components, thus providing both alignment simplicity and system stability. The reference arm was made of an integrated fibered delay-line spliced to two broadband 50:50 couplers. The chalcogenide fiber under-test was inserted into the test path by means of a face to face clived-fiber injection set-up ensured by micron precision holders. The resulting interference pattern was monitored in the frequency domain thanks to an optical spectrum analyzer (OSA). In order to measure the chromatic dispersion of our chalcogenide fibers on a wide range of wavelengths, we have first generated a supercontinuum spanning from $1100 \mathrm{~nm}$ to more than $1750 \mathrm{~nm}$ (OSA upper limit). The supercontinuum was obtained through the amplification and nonlinear propagation of a 10-ps pulse train into a highly nonlinear fiber (HNLF). More precisely, the picosecond-pulse train was delivered by a $22-\mathrm{MHz}$ modelocked fiber laser centered around $1553 \mathrm{~nm}$, amplified at an average power of $28 \mathrm{dBm}$ by means of an Erbium doped fiber amplifier and finally injected into a 500-m long HNLF from $O F S$ whose parameters at $1550 \mathrm{~nm}$ are a chromatic dispersion $\mathrm{D}=0.5 \mathrm{ps} / \mathrm{km} . \mathrm{nm}$, a dispersion slope $\mathrm{S}=0.01 \mathrm{ps} / \mathrm{km} . \mathrm{nm}^{2}$ and a nonlinear Kerr coefficient $\gamma=10.5 \mathrm{~W}^{-1} \cdot \mathrm{km}^{-1}$. The resulting supercontinuum is illustrated in Fig. 4a and shows a high spectral brightness allowing measurement from $1.1 \mu \mathrm{m}$ to 1.75 $\mu \mathrm{m}$. Figure $4 \mathrm{~b}$ (solid line) shows a typical interference pattern monitored around $1550 \mathrm{~nm}$ where a central fringe is clearly visible. The dispersion of the fiber sample in the 1.1-1.7 $\mu \mathrm{m}$ range was then obtained by monitoring the position of this central fringe as a function of the temporal delay $\Delta$ provided by the reference arm. Experimental results (circles) are plotted in Fig. 4c and are well fitted by a two order polynomial function (black solid line). The dispersion curve of our sample is then simply deduced from the delay variations by the following relation:

$$
D(\lambda)=\frac{1}{L c} \frac{d \Delta}{d \lambda}
$$

Figure $4 \mathrm{~d}$ shows the resulting dispersion curve of the $2 \mathrm{~S} 1 \mathrm{G} \_2$ chalcogenide fiber as a function of wavelength. The dispersion and dispersion slope value at $1550 \mathrm{~nm}$ were found to be $\mathrm{D}=$ $406 \mathrm{ps} / \mathrm{nm} / \mathrm{km}$ and $\mathrm{S}=d D / d \lambda=1.1 \mathrm{ps} / \mathrm{nm}^{2} / \mathrm{km}$. In order to validate our measurements, we have finally checked that the resulting dispersion values allowed us to retrieve the experimental interference pattern $I_{\text {int }}$ theoretically given by:

$$
I_{\mathrm{int}}=I_{1}^{2}+I_{2}^{2}+2 \sqrt{I_{1} I_{2}} \cos (\Phi)
$$

where $\Phi$ is the relative phase between the two arms of the interferometer calculated thanks to the following expression:

$\Phi=\Phi_{0}+\sum_{k=1}^{\infty} \frac{\left(\omega-\omega_{0}\right)^{k}}{k !}\left(\frac{d^{k} \beta^{C H A}}{d \omega^{k}} L^{C H A}-\frac{d^{k} \beta^{R E F}}{d \omega^{k}} L^{R E F}\right)$

In this expression, $\Phi_{0}$ is a constant phase difference. $\beta^{C H A}$ and $\beta^{R E F}$ are the mode-propagation constants of the chalcogenide fiber and reference fiber, respectively, whereas $\omega_{0}$ corresponds to the optical frequency for which the temporal delay 
mismatchs between the two arms vanishes. Figure $4 \mathrm{~b}$ (crosses) shows the theoretical interference spectrum obtained by reporting the value of D and S in expression (4) and (3). As can be seen, a good agreement is obtained between the experimental and theoretical spectra, thus validating the values of group-velocity dispersion and slope calculated from equation (2).

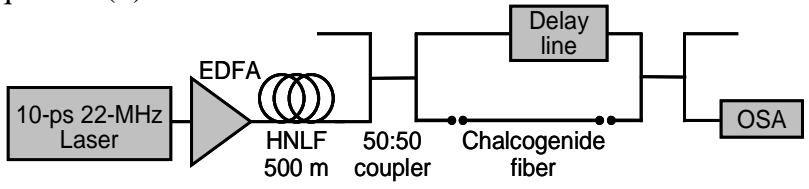

Fig. 3. Experimental set-up for chromatic dispersion characterization.
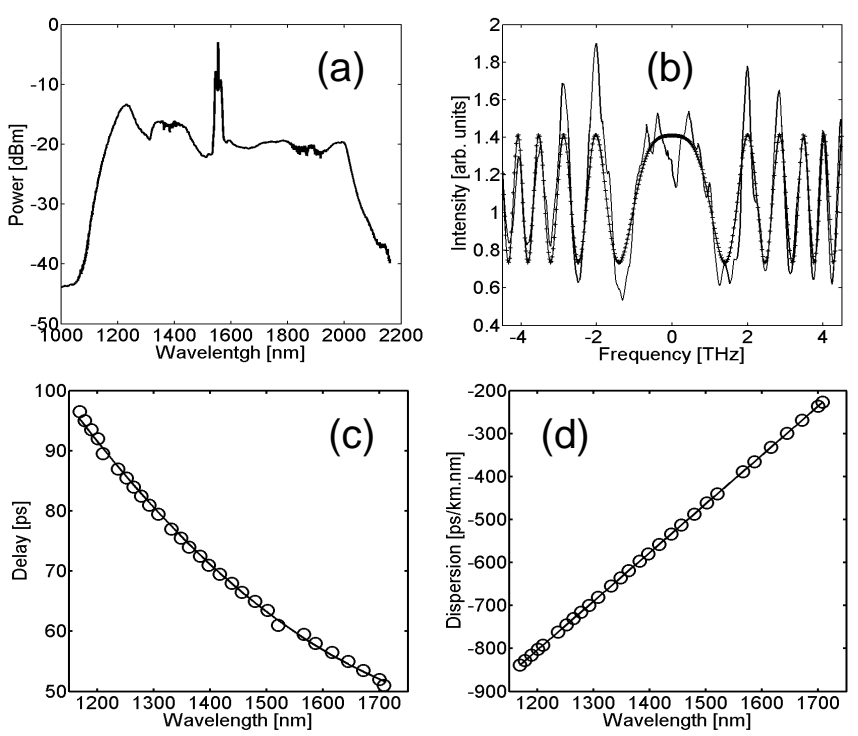

Fig. 4. (a) Supercontinuum generated at the output of the HNLF fiber (b) Interference fringes at the coupler output (c) Delay vs wavelength, experimental values for the GeSbS_2 fiber (circles), polynomial fit (solid line) (d) Dispersion vs wavelength, experimental values (circles), linear fit (solid line).

The same experiment was completed for all of the chalcogenide fibers and results at $1550 \mathrm{~nm}$ are indicated in the Table 2.

\section{BIREFRINGENCE}

The polarisation mode dispersion properties is an important parameter for any optical signal processing function since it could induce large pulse distortion or broadening [36]-[37]. In our work, the birefringence properties of the microstructured chalcogenide fibers were measured by means of the spectral interference based experimental set-up described in Fig. 5 [38]-[39]. An amplified spontaneous emission source (ASE) is passing through a polarizer ( $\mathrm{Pol}$ ) and is injected into the fiber under-test by means of the alignment option of a splicing device. At the output of the fiber, both slow and fast replicas of the signal interfere on an analyzer (A) before detection in the frequency domain thanks to an optical spectrum analyzer (OSA) [38]-[39].

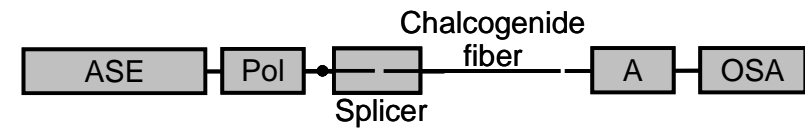

Fig. 5. Experimental set-up for birefringence characterization.

Due to the first order frequency dependence of state of polarization, a fringe-like spectrum is observed on the OSA at the output of the analyzer. More precisely, the intensity detected by the OSA is of the form [38]-[39]:

$$
I=a+b \cos (\Delta \beta(\omega) L)
$$

where $a$ and $b$ are constant coefficients which depend on the relative angle positions of input and output polarizer/analyzer. $L$ is the length of the fiber and $\Delta \beta$ the propagation constant difference between the slow and fast axes of the fiber which can be expanded into:

$$
\Delta \beta(\omega)=\Delta \beta\left(\omega_{0}\right)+\left(\frac{d \Delta \beta}{d \omega}\right)_{\omega_{0}} \Delta \omega,
$$

where $\Delta \omega=\omega-\omega_{0}$ is the frequency detuning with respect to the carrier frequency $\omega_{0}$ of the signal. The polarization mode dispersion of the fiber is then characterized by the differential group delay $(D G D)$ given by:

$$
D G D=\frac{1}{\operatorname{Vgx}\left(\omega_{0}\right)}-\frac{1}{\operatorname{Vgy}\left(\omega_{0}\right)}=\left(\frac{d \Delta \beta}{d \omega}\right)_{\omega_{0}},
$$

where $V g x$ and $V g y$ are the group velocities along the slow and fast axes, respectively.

We can see by injecting expressions (6) and (7) in equation (5) that the spectrum monitored on the OSA at the output of the analyzer consists of a periodic signal which fringe spacing $\Omega$ provides a direct measurement of the DGD thanks to the following relation:

$$
D G D=\frac{2 \pi}{\Omega L} .
$$

Figure 6 represents a typical spectrum recorded at the output of a $0.73-\mathrm{m}$ long GeSbS_1 chalcogenide fiber sample after optimization of input and output polarizer/analyzer angle positions maximizing the fringe contrast. We can see 15 interference periods leading to a DGD of $5.5 \mathrm{ps} / \mathrm{m}$.

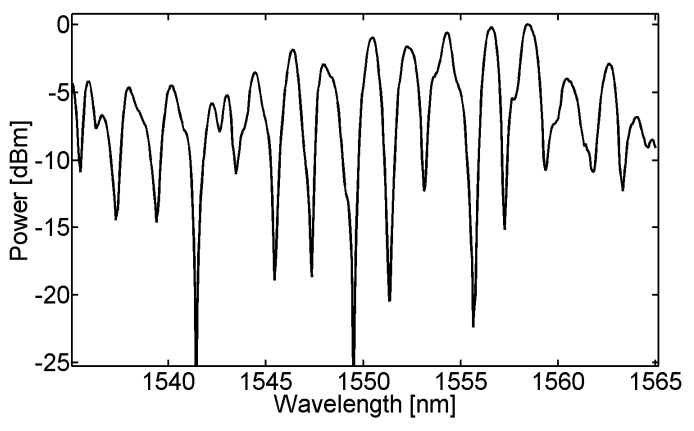

Fig. 6. Interference fringes at the chalcogenide GeSbS_1 fiber output.

Note that several irregularities appear in the interference fringes and arise from the fiber structure inhomogeneities. We have reported the experimental values of DGD in table 5 as well as the optical index difference between the two axes. 
Typical high values around $10^{-4}$ were found, close to typical values of standard silica polarization maintaining fibers. More importantly, we have found a dramatic stronger birefringence for the GeSbS_1 fiber compare to the GeSbS_2 sample, which originates from the core-size reduction occurring during the drawing process and underlines the practical issue to reach symmetric microstructures of small dimensions. Previous works have already reported similar analysis showing that small silica core PCFs (large air holes and small pitch) are more sensitive to fabrication-induced asymmetries in the microstruture giving rise to higher form of birefringence that makes the polarization maintaining fiber [40]-[42]. Experimental results obtained for the three microstructured chalcogenide fibers are summarized in Table 2.

\section{SElF-PhASE MODULATION}

\section{A. Experimental setup}

In this section, we have focused our attention on the nonlinear characterization of our chalcogenide fibers. We have studied the well-known self-phase modulation effect and compare our experimental results to numerical simulations so as to deduce the Kerr nonlinear coefficient. Figure 7 shows the experimental set-up employed to characterize the nonlinear Kerr properties of the microstructured chalcogenide fibers [19]-[20]. A mode-locked fiber laser generates 8.3-ps pulses at a repetition rate of $19.3 \mathrm{MHz}$ around $1556 \mathrm{~nm}$. The signal is then passing through a polarization controller (PC) adjusted to maximize the self-phase modulation (SPM) induced spectral broadening within the chalcogenide fiber. The signal power is controlled by a variable attenuator (VA) and measured by a power-meter (PwM) before injection into the fiber under-test. Note that the input signal was coupled into the chalcogenide fiber by means of the alignment option of an optical fiber splicing device, which permits an efficient fiber coupling with losses around $2.8 \mathrm{~dB}$. At the output of the fiber, the signal is finally monitored thanks to an optical spectrum analyzer (OSA).

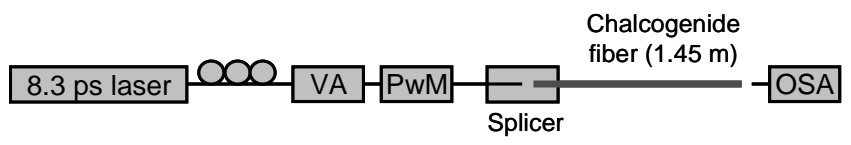

Fig. 7. Experimental set-up for self-phase modulation characterization.

\section{B. Experimental results}

Figure 8a illustrates the spectra recorded at the output of the 1.45-m long GeSbS_1 chalcogenide fiber as a function of the input average power $\left(P_{0}\right)$. The values of $P_{0}$ are obtained by means of the power-meter with a subtraction to the couplingloss in the splicer. Experimental results exhibit a typical selfphase modulation behavior with spectral broadening and oscillations. An asymmetry can also be observed in the output spectra, which we attribute to the initial asymmetry spectrum of our picosecond laser source. In order to determine the Kerr coefficient of the GeSbS_1 chalcogenide fiber, we have completed numerical simulations and compared our numerical predictions to the experimental results of Fig. 8a. The numerical evolution of pulses was obtained by means of a split-step Fourier algorithm including experimental data of chromatic dispersion and losses. Results are plotted in Fig. 8b and show a good agreement with experimental results of Fig. 8a. From these simulations, we deduced a nonlinear Kerr coefficient of $\gamma=517 \mathrm{~W}^{-1} \mathrm{~km}^{-1}$ which is 400 times larger than a conventional single mode fused silica fiber (SMF). The same experiment was done in the GeSbS_2 chalcogenide fiber for which the electric field is less confined by the microstructure $\left(A_{e f f}=50 \mu \mathrm{m}^{2}\right)$ than in the GeSbS_1 fiber $\left(A_{e f f}=22 \mu \mathrm{m}^{2}\right)$. As previously, the nonlinear Kerr coefficient of the GeSbS_2 fiber was deduced from the comparison between the experimental and numerical spectra and was found to be $\gamma=$ $227 \mathrm{~W}^{-1} \mathrm{~km}^{-1}$.
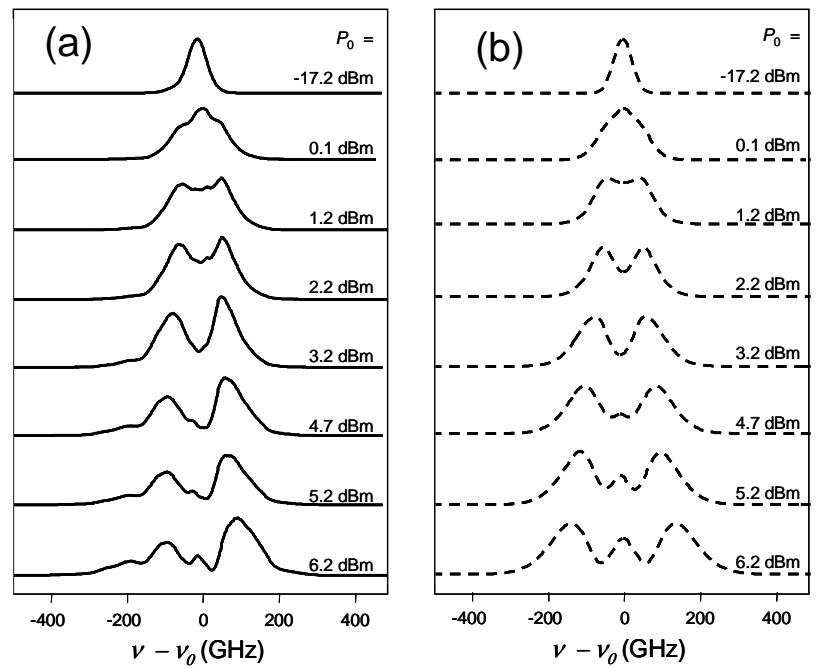

Fig. 8. (a) Experimental results: output spectra for the 1.45-m long GeSbS_1 chalcogenide fiber as a function of input power (b) Numerical simulations corresponding to results of Fig. a.

Figure 9 shows the experimental results obtained for the AsSe $21-\mu \mathrm{m}^{2}$ chalcogenide fiber.

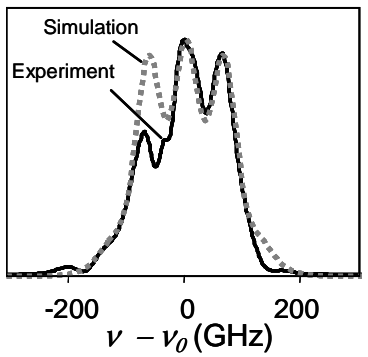

Fig. 9. Output spectrum for the 1.15-m long AsSe chalcogenide fiber for an input average power of $1 \mathrm{~mW}$, dashed-line: corresponding numerical simulation.

Results are similar to those of Fig. 8 and show a good agreement between experiment and simulation. Numerical simulations provide a good estimation of the nonlinear Kerr coefficient, which was found to be $\gamma=2000 \mathrm{~W}^{-1} \mathrm{~km}^{-1}$. One can however notice, in both Figs. 8 and 9, that the central part of the spectrum exhibits some ripples and that some spectral pedestals have developed in the spectrum. Those features 
outline the strong interaction between self-phase modulation and the high dispersion of the AsSe fiber, leading to the development of the wave-breaking phenomenon [43]. Finally, the nonlinear Kerr coefficient $\gamma$ and nonlinear index $n_{2}$ obtained for the three microstructured chalcogenide fibers are indicated in Table 2 and are quite close to the values mentioned in the literature [44]-[46], 2.4.10 $0^{-17} \mathrm{~m}^{2} / \mathrm{W}$ for $\mathrm{As}_{2} \mathrm{Se}_{3}$ in [45] and $2.10^{-18} \mathrm{~m}^{2} / \mathrm{W}$ for $\mathrm{Ge}_{23} \mathrm{Sb}_{12} \mathrm{~S}_{65}$ in [46]. Note that $n_{2}$ was calculated from the Kerr coefficient following the relation [29]:

$$
n_{2}=\frac{\gamma \lambda A_{\text {eff }}}{2 \pi}
$$

It's important to note that the self-phase modulation induced spectrum broadening is here only limited by the effective length of the fiber $(0.7 \mathrm{~m}$ for the GeSbS_1 PCF) and not by the dispersion length $(40 \mathrm{~m})$, thus underlining the critical issue of linear losses.

\begin{tabular}{|c|c|c|c|}
\hline Parameter & \multicolumn{3}{|c|}{ Fiber } \\
\hline & GeSbS_1 & GeSbS_2 & AsSe \\
\hline$\Lambda(\mu \mathrm{m})$ & 9 & 13.25 & 7 \\
\hline$d / \Lambda$ & 0.31 & 0.31 & 0.42 \\
\hline$A_{\text {eff }}\left(\mu \mathrm{m}^{2}\right)$ & 22 & 50 & 21 \\
\hline$\alpha(d B / \mathrm{m})$ & 5 & 5.5 & 10 \\
\hline$D(\mathrm{ps} / \mathrm{nm} / \mathrm{km})$ & -421 & -406 & -760 \\
\hline$S\left(\mathrm{ps} / \mathrm{nm}^{2} / \mathrm{km}\right)$ & 0.9 & 1.1 & 2.7 \\
\hline$D G D(\mathrm{ps} / \mathrm{m})$ & 5.5 & 0.8 & 1.24 \\
\hline$\Delta n$ & $1.610^{-3}$ & $2.410^{-4}$ & $3.710^{-4}$ \\
\hline$\gamma\left(\mathrm{W}^{1} \mathrm{~km} \mathrm{~m}^{-1}\right)$ & 517 & 227 & 2000 \\
\hline$n_{2}\left(\mathrm{~m}^{2} / \mathrm{W}\right)$ & $2.810^{-18}$ & $2.810^{-18}$ & $1.110^{-17}$ \\
\hline
\end{tabular}

Table 2: Measured optical properties of the three microstructured chalcogenide fibers.

\section{BRILLOUIN SCATTERING EFFECT}

The Brillouin scattering effect in step-index chalcogenide fibers has already been investigated in previous works [18], [23]-[25]. The Brillouin gain was measured to be more than 150 times larger than fused silica fiber [18], [23]-[25] which enables a high degree of integration in a host of applications such as amplification of a small optical signal [25], fiber-based lasing systems [25] or tunable delay lines via slow and fast light processes [18]. In this section, we present the experimental characterization of the Brillouin scattering occurring in the 2-m long GeSbS_2 microstructured chalcogenide fiber [17]. Figure 10 illustrates the experimental set-up. A continuous wave (cw) is first generated at $1552 \mathrm{~nm}$ by means of a distributed feedback (DFB) laser diode having a spectral linewidth given by the manufacturer of $150 \mathrm{kHz}$. An acousto-optical switch (AO) converts this cw into a 140-ns quasi Gaussian pulse train at a repetition rate of $500 \mathrm{kHz}$. The pulse train is then amplified by means of an EDFA at an average power of $30 \mathrm{dBm}$. A variable attenuator (VA), associated with a 90:10 coupler and a power-meter (PwM), is then used to adjust the injected average power into the fiber. Finally, the resulting signal is launched into a circulator whose port \#2 is used to inject the incident light into the fiber undertest by means of a splicing device and port \#3 to collect the backscattered light. The intensity of the Brillouin Stokes component is then measured at port \#3 thanks to an optical spectrum analyzer (OSA) having a spectral resolution of 0.07 $\mathrm{nm}$.

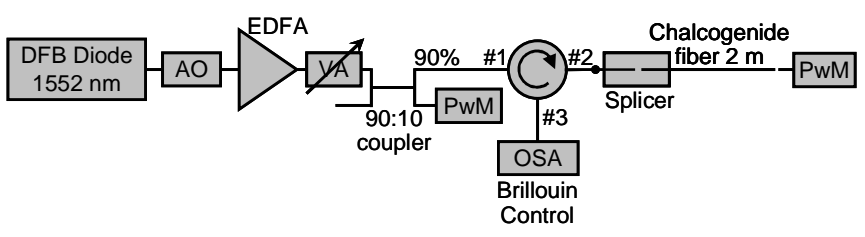

Fig. 10. Experimental set-up for Brillouin Characterization.

Inset in Fig. 11 shows a typical backscattered optical spectrum obtained at port \#3 of the circulator. A high power backscattered Stokes Brillouin component is localized around $-8.2 \mathrm{GHz}$ which is close to the $-7.95 \mathrm{GHz}$ value obtained by Abedin in a single mode $\mathrm{As}_{2} \mathrm{Se}_{3}$ fiber [23] and much lower than in a classical fused silica fiber $(11 \mathrm{GHz})$ [29]. In order to determine the threshold and gain of the Brillouin scattering, we have illustrated in Fig. 11 the transmitted and backscattered Brillouin component powers as a function of the injected power. We can clearly observe a typical Brillouin behaviour with an exponential growth of the amount of energy backscattered from the fiber as well as a quasi saturation of the transmitted power. The Brillouin threshold $P_{t h}$, usually defined as the power leading to an amount of backscattered energy equal to the transmitted one [29], corresponds here to a threshold value of $1.95 \mathrm{~W}$ while the Brillouin gain $g_{B}$ is then calculated by means of the following relation [29]:

$$
g_{B} P_{t h} L_{e f f} / A_{e f f} \approx 21,
$$

which corresponds to a Brillouin gain of $g_{B}=8.10^{-10} \mathrm{~m} / \mathrm{W}$ that is to say 100 more than in a standard silica fiber [17]. This value is smaller than those reported in refs. [36] and [24] but could be explained by the definition of the Brillouin threshold used in these references. Finally, we have also completed the measurements by determining the linewidth of the Brillouingain by means of an auto-heterodyne technique [17]. The Brillouin bandwidth was found to be $9.5 \mathrm{MHz}$ (vs $13 \mathrm{MHz}$ for standard silica fiber) which is slightly smaller than in ref. [36], presumably due to the different glass composition and the presence of the microstructure.

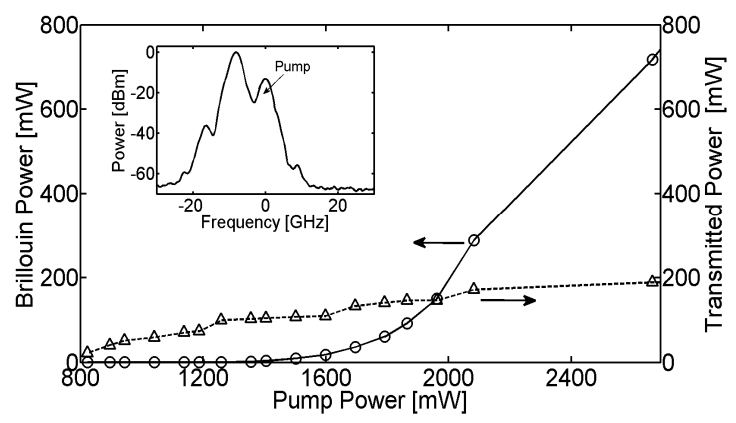

Fig. 11. Inset: Experimental Brillouin spectrum recorded at port \#3 of the circulator for the 2-m long GeSbS_2 chalcogenide fiber. Backscattered and transmitted powers as a function of the input power Chalcogenide fiber. 


\section{RAMAN SCATTERING EFFECT}

In this section, we have focused our attention on the Raman scattering effect occurring in the GeSbS_2 microstructured chalcogenide fiber [17]. The strong nonlinear feature of the Raman process in chalcogenide fibers was previously underlined through the third order cascaded Raman wavelength generation occurring in a step-index $\mathrm{As}_{2} \mathrm{~S}_{3}$ fiber [26]. In this last work, Kulkami and co-workers have determined the Raman gain coefficient, which was found as large as $\sim 89$ times higher than fused silica [26]. The strong efficiency of Raman effect was also employed in order to design a Chalcogenide glass Raman fiber laser [27]. In another hand, very recently, a numerical study by Varshney et al. has also shown that As-Se photonic crystal fibers could increase the Raman gain efficiency by a factor four compare to conventional As-Se fibers [28], thus underlining the practical interest of this kind of fiber for Raman applications.

Figure 12 shows the experimental set-up used to characterize the Raman effect occurring in the GeSbS_2 microstructured chalcogenide fiber. A $1-\mathrm{kHz} 10-\mathrm{ns}$ square pulse laser emitting around $1553 \mathrm{~nm}$ is used as Raman pump. In order to determine the Raman gain, we have measured the amplification undergone by a seed-signal injected in a copropagating configuration and shifted from the Raman pump by $83 \mathrm{~nm}$ for the chalcogenide fiber (see below). The 1636-nm seed component was obtained through the generation of a broad frequency-comb via the propagation of an initial 30$\mathrm{dBm}$ beat signal centred around $1555 \mathrm{~nm}$ into a $500-\mathrm{m}$ long highly nonlinear fiber (HNLF) [47]-[49]. Note that, at the output of the fiber under-test, only the part of the signal wave which has been amplified was injected into the optical spectrum analyzer thanks to an acousto-optic synchronised on the Raman pump.

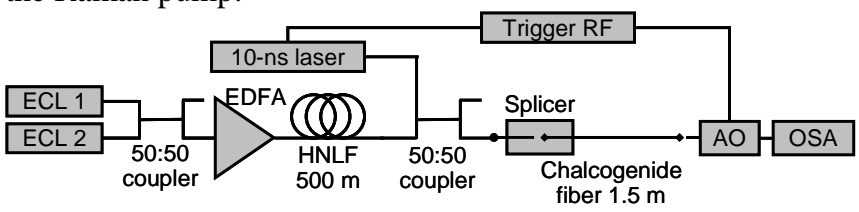

Fig. 12. Experimental set-up for Raman characterization.

Figure 13a illustrates the spontaneous Raman response (in absence of seeding) occurring into the chalcogenide fiber for an input pump power of $80 \mathrm{~W}$. The Raman detuning was measured at $83 \mathrm{~nm}(9.7 \mathrm{THz})$ with a typical FWHM of $5.5 \mathrm{~nm}$. In the stimulated regime, we have measured the output power of the 1636-nm amplified signal as a function of the input Raman pump power $P_{\text {pump }}$. As can be seen in Fig. 13b, a strong amplification of the input signal was observed with a maximum gain close to $G=37 \mathrm{~dB}$. This value corresponds to a gain per unit length of $24.7 \mathrm{~dB} / \mathrm{m}$ and a Raman gain of $g_{R}=\ln (G) . A_{\text {eff }} / P_{\text {pump. }} \cdot L_{\text {eff }}=1.8 .10^{-11} \mathrm{~m} / \mathrm{W}$, that is to say 180 times larger than a fused silica fiber [17] and three-fold higher than the previous result reported in ref. [26]. Finally, the inset in Fig. 13b illustrates a typical amplified signal spectrum recorded for a pump power of $P_{\text {pump }}=24.5 \mathrm{~W}$.
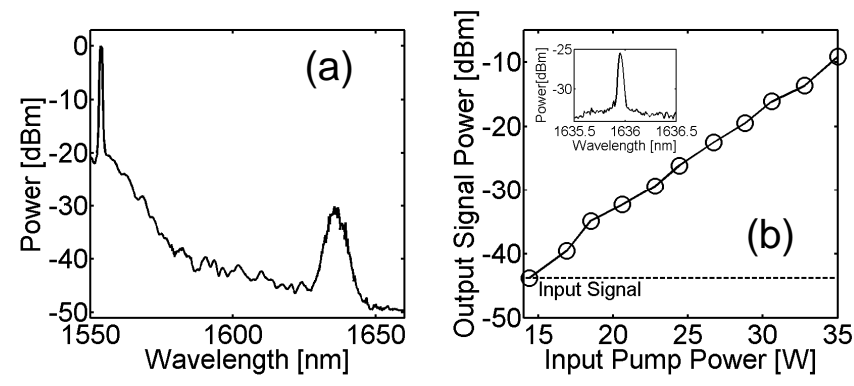

Fig. 13. (a) Spontaneous Raman scattering at the output of the GeSbS_2 chalcogenide fiber for an input pump power of $80 \mathrm{~W}$. (b) Output signal power as a function of input pump power for the chalcogenide fiber. Inset: Output amplified signal spectrum for a pump power of $24.5 \mathrm{~W}$.

\section{COnClusion}

In this paper, we have investigated the linear and nonlinear properties of GeSbS and AsSe chalcogenide microstructured optical fibers. The manufactured fibers were found to have a nonlinear coefficient two order of magnitude higher than standard silica fibers as well as a Brillouin and Raman gain 100 and 180 larger than fused silica, respectively.

In order to enlarge the field of applications of this kind of fiber, for example so as to generate supercontinuum or signal processing device around $1550 \mathrm{~nm}$, a careful design of the microstructure has to be performed in order to shift the zero dispersion wavelength in the $\mathrm{C}$ band, losses should be reduced thanks to a dramatic purification of the glasses whereas nonlinearity could be still enhanced by reducing the size of the core. Finally, we believe that if dimensions of the fiber are suitably designed and manufactured, chalcogenide fibers could find many applications in nonlinear optics and would be a promising candidate for ultra compactness nonlinear devices.

\section{ACKNOWLEDGMENTS}

We would like to acknowledge financial support of the Agence National de la Recherche (FUTUR project). We thank P. Grelu and G. Millot from the Institut Carnot de Bourgogne for fruitful discussions on chromatic dispersion and birefringence measurements. We also thank $\mathrm{M}$. Thual and $\mathrm{Y}$. Dumeige from ENSSAT of Lannion for their help on selfphase modulation measurements.

\section{REFERENCES}

[1] J. C. Knight, T. A. Birks, P. S. J. Russell, and D. M. Atkin, “All-silica single-mode optical fiber with photonic crystal cladding," Opt. Lett., vol. 21, pp. 1547-1549, 1996.

[2] P. S. Russell, "Photonic crystal fibers," Science, vol. 299, pp. 358-362, 2003.

[3] J. M. Dudley, G. Genty, and S. Coen, "Supercontinuum generation in photonic crystal fiber,” Rev. Mod. Phys., vol. 78, pp. 1135-1184, 2006.

[4] I. Hartl, X. D. Li, C. Chudoba, R. K. Ghanta, T. H. Ko, J. G. Fujimoto, J. K. Ranka, and R. S. Windeler, "Ultrahigh-resolution optical coherence tomography using continuum generation in an air-silica microstructure optical fiber," Opt. Lett., vol. 26, pp. 608-610, 2001.

[5] S. A. Diddams, D. J. Jones, J. Ye, T. Cundiff, J. L. Hall, J. K. Ranka, R. S. Windeler, R. Holzwarth, T. Udem, and T. W. Hanch, "Direct link between microwave and optical frequencies with a $300 \mathrm{THz}$ femtosecond laser comb," Phys. Rev. Lett., vol. 84, pp. 5102-5105, 2000 . 
[6] P. Petropoulos, T. M. Monro, W. Belardi, K. Furusawa, J. H. Lee, and D. J. Richardson, " $2 R$-regenerative all-optical switch based on a highly nonlinear holey fiber," Opt. Lett., vol. 26, pp. 1233-1235, 2001.

[7] F. Benabid, J. Knight, and P. Russell, "Particle levitation and guidance in hollow-core photonic crystal fiber," Opt. Express, vol. 10, pp. 1195 1203, 2002.

[8] L. B. Shaw, P. A. Thielen, F. H. Kung, V. Q. Nguyen, J. S. Sanghera and I. D. Aggarwal, "IR Supercontinuum Generation in As-Se Photonic Crystal Fiber," in Proc. Optical Fiber Communications Conference (OFC 2007), Anaheim USA, March 2007, TuC5.

[9] N. Sugimoto, T. Nagashima, T. Hasegawa, S. Ohara, K. Taira, and K. Kikuchi, "Bismuth-based optical fiber with nonlinear coefficient of $1360 \mathrm{~W}^{-1} \mathrm{~km}^{-1}$," in Proc. Optical Fiber Communications Conference (OFC2004), Anaheim USA, March 2004, PDP26 (Postdeadline paper).

[10] J. H. V. Price, T. M. Monro, H. Ebendorff-Heidepriem, F. Poletti, P. Horak, V. Finazzi, J. Y. Y. Leong, P. Petropoulos, J. C. Flanagan, G. Brambilla, M. Feng and D. J. Richardson, "Mid-IR Supercontinuum Generation from Nonsilica Microstructured Optical Fibers,” IEEE J. Sel. Top. Quantum Electron., vol. 13, pp. 738-749, 2007.

[11] P. Domachuk, N. A. Wolchover, M. Cronin-Golomb, A. Wang, A. K. George, C. M. B. Cordeiro, J. C. Knight, and F. G. Omenetto, "Over $4000 \mathrm{~nm}$ bandwidth of mid-IR supercontinuum generation in subcentimeter segments of highly nonlinear tellurite PCFs," Opt. Express vol. 16, pp. 7161-7168, 2008.

[12] T. Nagashima, T. Hasegawa, S. Ohara, and N. Sugimoto, "Dispersion shifted Bi2O3-based photonic crystal fiber," in Proc. European Conference on Optical Communication (ECOC 2008), Cannes France 2008, We.1.3.3.

[13] D. Pelusi, F. Luan, E. Magi, M. R. Lamont, D. J. Moss, B. J. Eggleton, J. S. Sanghera, L. B. Shaw, and I. D. Aggarwal, "High bit rate all-optical signal processing in a fiber photonic wire," Opt. Express, vol. 16, pp. 11506-11512, 2008

[14] D. Yeom, E. C. Mägi, M. R. E. Lamont, M. A. F. Roelens, L. Fu, and B. J. Eggleton, "Low-threshold supercontinuum generation in highly nonlinear chalcogenide nanowires," Opt. Lett., vol. 33, pp. 660-662, 2008.

[15] M. Pelusi, V. G. Ta'eed, L. Fu, E. C. Mägi, M. R. E. Lamont, S. Madden, D.-Y. Choi, D. A. P. Bulla, B. Luther-Davies, and B. J. Eggleton, "Applications of highly-nonlinear chalcogenide glass devices tailored for high-speed all-optical signal processing," IEEE J. Sel. Top. Quantum Electron., vol. 14, pp. 529-539, 2008.

[16] P. Petropoulos, H. Ebendorff-Heidepreim, V. Finazzi, R. C. Moore, K. Frampton, D. J. Richardson, and T. M. Monro, "Highly nonlinear and anomalously dispersive lead silicate glass holey fibers," Opt. Express, vol. 11, pp. 3568-3573, 2003.

[17] C. Fortier, J. Fatome, S. Pitois, F. Smektala, G. Millot, J. Troles, F. Desevedavy, P. Houizot, L. Brilland, and N. Traynor, "Experimental investigation of Brillouin and Raman scattering in a 2SG sulfide glass microstructured chalcogenide fiber," Opt. Express, vol. 16, pp. 9398 9404, 2008

[18] K. Y. Song, K. S. Abedin, K. Hotate, M. González Herráez, and L. Thévenaz, "Highly efficient Brillouin slow and fast light using $\mathrm{As}_{2} \mathrm{Se}_{3}$ chalcogenide fiber," Opt. Express, vol. 14, pp. 5860-5865, 2006.

[19] L. B. Fu, M. Rochette, V. G. Ta'eed, D. J. Moss, and B. J. Eggleton, "Investigation of self-phase modulation based optical regeneration in single mode As2Se3 chalcogenide glass fiber," Opt. Express, vol. 13, pp. 7639-7646, 2005.

[20] L. Fu, V. G. Ta'eed, E. C. Mägi, I. C. M. Littler, M. Pelusi, M. R. E. Lamont, A. Fuerbach, H. C. Nguyen, D. I. Yeom, and B. J. Eggleton, "Highly nonlinear chalcogenide fibres for all-optical signal processing," Optical and Quantum Electronics, vol. 39, pp. 1115-1131, 2008.

[21] L. Brilland, F. Smektala, G. Renversez, T. Chartier, J. Troles, T. N. Nguyen, N. Traynor, and A. Monteville, "Fabrication of complex structures of Holey Fibers in chalcogenide glasses," Opt. Express, vol 14, pp. 1280-1285, 2006.

[22] L. Brilland, J. Troles, P. Houizot, F. Désévédavy, Q. Coulombier, G. Renversez, T. Chartier, T. N. Nguyen, J. L. Adam, N. Traynor, "Interfaces impact on the transmission of chalcogenides photonic crystal fibres;" J. of The Ceramic Society of Japan, vol. 116, pp 1024-1027, 2008
[23] K. S. Abedin, "Observation of strong stimulated Brillouin scattering in single-mode As2Se3 chalcogenide fiber," Opt. Express, vol. 13, pp. 10266-10271, 2005.

[24] C. Florea, M. Bashkansky, Z. Dutton, J. Sanghera, P. Pureza, and I. Aggarwal, "Stimulated Brillouin scattering in single-mode As2S3 and As2Se3 chalcogenide fibers," Opt. Express, vol. 14, pp. 12063-12070, 2006.

[25] K. S. Abedin, "Brillouin amplification and lasing in a single-mode As2Se3 chalcogenide fiber,” Opt. Lett., vol. 31, pp. 1615-1617, 2006.

[26] O. P. Kulkarni, C. Xia, D. J. Lee, M. Kumar, A. Kuditcher, M. N. Islam, F. L. Terry, M. J. Freeman, B. G. Aitken, S. C. Currie, J. E. McCarthy, M. L. Powley, and D. A. Nolan, "Third order cascaded Raman wavelength shifting in chalcogenide fibers and determination of Raman gain coefficient," Opt. Express, vol 14, pp. 7924-7930, 2006.

[27] S. D. Jackson and G. Anzueto-Sanchez, "Chalcogenide glass Raman fiber laser,” Appl. Phys. Lett., vol. 88, pp. 221106, 2006.

[28] S. K. Varshney, K. Saitoh, K. Iizawa, Y. Tsuchida, M. Koshiba, and R. K. Sinha, "Raman amplification characteristics of As2Se3 photonic crystal fibers," Opt. Lett., vol. 33, pp. 2431-2433, 2008.

[29] G. P. Agrawal, Nonlinear Fiber Optics, 3th Ed, Academic Press, Boston, 2001

[30] G. G. Devyatyk, M. F. Churbanov, I. V. Schripatchev, G. E. Snopatin, E. M. Dianov, V. G. Plotichenko, "Recent developpement in As-S glass fibres," Journal of non crystalline solids, vol. 256 \& 257, pp. 318-322, 1999.

[31] J. Troles, L. Brilland, F. Smektala, P. Houizot, F. Desevedavy, C. Q., N. Traynor, T. Chartier, T. N. Nguyen, J. L. Adam, and G. Renversez, "Chalcogenide Microstructured Fibers for Infrared Systems, Elaboration Modelization, and Characterization," Fiber and integrated optics, vol. 28, pp. 11-26, 2009.

[32] H. Chen, "Simultaneous measurements of non-linear coefficient, zerodispersion wavelength and chromatic dispersion in dispersion-shifted fibers by four-wave mixing", Opt. Commun., vol. 220, pp. 331-335, 2003.

[33] J. Fatome, S. Pitois, and G. Millot, "Measurement of nonlinear and chromatic dispersion parameters of optical fibers using modulation instability," Opt. Fiber Technol., vol. 12, pp. 243-250, 2006.

[34] T. N. Nguyen, T. Chartier, M. Thual, P. Besnard, L. Provino, A. Monteville, and N. Traynor, "Simultaneous measurement of anomalous group-velocity dispersion and nonlinear coefficient in optical fibers using soliton-effect compression," Opt. Commun., vol. 278, pp. 60-65, 2007.

[35] F. Koch, S. V. Chernikov, and J. R. Taylor, "Dispersion measurement in optical fibers over the entire spectral range from $1.1 \mu \mathrm{m}$ to $1.7 \mu \mathrm{m}$ ", Opt. Commun., vol 175, pp. 209-213, 2000.

[36] J.P. Gordon, and H. Kogelnik, "PMD fundamentals: Polarization mode dispersion in optical fibers," PNAS 97, pp. 4541-4550, 2000.

[37] J. Garnier, J. Fatome, and G. Le Meur, "Statistical analysis of pulse propagation driven by polarization-mode dispersion," J. Opt. Soc. Am. B, vol. 19, pp. 1968-1977, 2002.

[38] X. D. Cao and D. D. Meyerhofer, "Frequency-domain interferometer for measurement of the polarization mode dispersion in single-mode optical fibers", Opt. lett., vol. 19, pp. 1837-1839, 1994.

[39] G. Millot and J. M. Dudley, "Polarization mode dispersion measurement in high-birefringence fibers by means of stimulated Raman scattering", Appl. Opt., vol. 41, pp. 2589-2591, 2002.

[40] I. K. Hwang, Y. J. Lee, and Y. H. Lee, "Birefringence induced by irregular structure in photonic crystal fiber," Opt. Express, vol. 11, pp. 2799-2806, 2003.

[41] K. L. Reichenbach and C. Xu, "The effects of randomly occurring nonuniformities on propagation in photonic crystal fibers," Opt. Express, vol. 13, pp. 2799-2807, 2005.

[42] L. Labonte, D. Pagnoux, P. Roy, F. Bahloul, and M. Zghal, "Numerical and experimental analysis of the birefringence of large air fraction slightly unsymmetrical holey fibres," Opt. Commun., vol. 262, pp. 180$187,2006$.

[43] C. Finot, B. Kibler, L. Provost, and S. Wabnitz, "Beneficial impact of wave-breaking or coherent continuum formation in normally dispersive nonlinear fibers,” J. Opt. Soc. Am. B, vol. 25, pp. 1938-1948, 2008.

[44] T. Cardinal, K. A. Richardson, H. Shim, A. Schulte, R. Beatty, K. Le Foulgoc, C. Meneghini, J. F. Viens and A. Villeneuve, "Non-linear 
optical properties of chalcogenide glasses in the system As-S-Se," J. Non-Cryst. Solids, vol. 256 \& 257, pp. 353-360, 1999.

[45] R. E. Slusher, G. Lenz, J. Hodelin, J. Sanghera, L. B. Shaw, and I. D. Aggarwal, "Large Raman gain and nonlinear phase shifts in high-purity As2Se3 chalcogenide fibers", JOSA B, vol. 21, pp. 1146-1155, 2004.

[46] L. Petit, N. Carlie, K. Richardson, A. Humeau, S. Cherukulappurath, and G. Boudebs, "Nonlinear optical properties of glasses in the system Ge/Ga-Sb-S/Se", Opt Lett., vol. 31, pp. 1495-1497, 2006.

[47] C. Fortier, B. Kibler, J. Fatome, C. Finot, S. Pitois, and G. Millot, "Allfibered high-quality low duty-cycle $160-\mathrm{GHz}$ femtosecond pulse source," Laser Phys. Lett., vol. 5, pp. 817-820, 2008.

[48] C. Finot, J. Fatome, S. Pitois, and G. Millot, "All-fibered high-quality low duty-cycle $20-\mathrm{GHz}$ and $40-\mathrm{GHz}$ picosecond pulse sources," IEEE Photon. Technol. Lett., vol. 19, pp. 1711-1713, 2007.

[49] J. Fatome, S. Pitois, and G. Millot, "320/640 GHz high-quality pulse sources based on multiple four-wave mixing in highly nonlinear optical fibre", Electron. Lett., vol. 41, pp. 1391-1392, 2005. 